\title{
Learning through guided imagery: A comprehensive teachers' guide for a balanced approach in preschool settings
}

\author{
Yi Hao \\ Northeastern Illinois University, United State
}

\begin{abstract}
While guided imagery has been widely used in the medical arena as a therapeutic tool and practiced as a teaching technique in elementary and secondary schools, little is known, however, about its effectiveness as a learning-teaching strategy in preschool settings. This study, by creating a curriculum model with the use of guided imagery in preschool settings, will fill the gap. It will equip teachers with an alternative teaching strategy that is creative, meaningful, and effective. Furthermore, since this model greatly encourages imagination, it will interest children and nurture the multimodal nature of young children's learning. Finally, the provision of this curriculum guide will increase classroom's right-brain learning activities and therefore maximize the brain power.
\end{abstract}

Keywords: guided imagery, imagination, teaching strategies, right-brain activity, Early Childhood 